\title{
Metformin as an initial adjunct to low-dose liraglutide enhances the weight-decreasing potential of liraglutide in obese polycystic ovary syndrome: Randomized control study
}

\author{
MOJCA JENSTERLE ${ }^{1}$, KATJA GORICAR ${ }^{2}$ and ANDREJ JANEZ ${ }^{1}$ \\ ${ }^{1}$ Department of Endocrinology, Diabetes and Metabolic Diseases, University Medical Centre; \\ ${ }^{2}$ Institute of Biochemistry, Faculty of Medicine, University of Ljubljana, SI-1000 Ljubljana, Slovenia
}

Received July 27, 2015; Accepted December 8, 2015

DOI: $10.3892 / \mathrm{etm} .2016 .3081$

\begin{abstract}
Liraglutide (LIRA) treatment is associated with the dose-dependent reduction of weight. Higher doses are more effective than lower doses, although higher doses are also more poorly tolerated. Metformin may enhance the weight-lowering potential of LIRA via the stimulatory modulation of incretin in addition to its direct beneficial effects in PCOS. The aim of the present study was to evaluate whether metformin as an adjunct to low-dose LIRA affects body weight with increased efficacy compared with low-dose LIRA alone in obese patients with PCOS. In a 12-week study, 44 obese women with PCOS were randomly offered either combined treatment (COMBO) with 1,000 mg metformin twice a day and $1.2 \mathrm{mg}$ LIRA once a day, or treatment with $1.2 \mathrm{mg}$ LIRA alone. The primary outcome of treatment was an alteration in the levels of obesity. A total of 43 patients [aged $30.3 \pm 4.4$ years; body mass index (BMI) $37.2 \pm 4.5 \mathrm{~kg} / \mathrm{m}^{2}$; mean \pm standard deviation] completed the study. The subjects treated with COMBO lost on average $6.2 \pm 2.4 \mathrm{~kg}$ compared with a $3.8 \pm 3.5 \mathrm{~kg}$ weight loss in the patients treated with LIRA alone $(\mathrm{P}=0.024)$. The BMI decreased by $2.2 \pm 0.8 \mathrm{~kg} / \mathrm{m}^{2}$ in patients treated with COMBO and by $1.4 \pm 1.2 \mathrm{~kg} / \mathrm{m}^{2}$ in patients treated with LIRA alone $(\mathrm{P}=0.024)$. A clinically significant $\geq 5 \%$ weight reduction was achieved in $59.1 \%$ of patients treated with COMBO and $42.9 \%$ of patients treated with LIRA alone. Reductions in glucose levels following oral glucose tolerance testing, as well as in androstenedione levels in the COMBO group were significantly greater compared with those in the LIRA group. The side effects were mild and transient in the two treatment groups. A combination of metformin and low-dose LIRA was
\end{abstract}

Correspondence to: Professor Andrej Janez, Department of Endocrinology, Diabetes and Metabolic Diseases, University Medical Centre, University of Ljubljana, Zaloška 7, SI-1000 Ljubljana, Slovenia

E-mail: andrej.janez@kclj.si

Key words: low-dose liraglutide, metformin, obesity, weight loss, polycystic ovary syndrome more effective than low-dose LIRA alone in reducing body weight in obese patients with PCOS.

\section{Introduction}

Obesity is not intrinsically associated with PCOS, yet the risk of obesity is $\geq 2.8$-fold higher in women with PCOS, compared with women without PCOS, with an estimated prevalence of $49 \%$ (1). The quantity and distribution of fat is a major contributor to the severity of PCOS $(2,3)$. Weight reduction in the individuals with the highest weight should be an important treatment target when PCOS is associated with obesity (4-6). However, weight-independent treatment targets that are intrinsically associated with the syndrome should also be involved in initial treatment of obese patients with PCOS. Recent clinical practice guidelines recommend lifestyle modification as the first line of intervention (7) in obese patients with PCOS, although the treatment goals with lifestyle intervention are usually challenging to achieve, and non-sustainable in everyday life.

The glucagon-like peptide-1 (GLP-1) receptor agonist liraglutide (LIRA) at a dose of $3 \mathrm{mg}$ daily was recently approved as an anti-obesity drug. Randomized placebo-controlled and active-comparator studies have demonstrated that LIRA is able to increase weight loss among overweight and obese patients in a dose-dependent manner with once-daily doses of 1.2-3.0 mg $(8,9)$. The proportion of patients experiencing 5-10\% weight loss was higher following treatment with LIRA compared with placebo and orlistat treatment (9). Higher doses of $\geq 3 \mathrm{mg}$ daily are more effective compared with lower doses, although this increase in efficacy occurs with decreased tolerability, a higher frequency of side effects and higher costs (10). These data support the potential benefit of LIRA among overweight and obese patients with prediabetes.

The efficacy of LIRA in obese women with PCOS has yet to be elucidated. A small randomized study conducted on treatment naïve obese women with PCOS demonstrated no significant difference between metformin and low-dose LIRA treatment following the measurement of patient obesity, metabolic and androgen profiles (11). However, the study also investigated a more severely obese subgroup, which showed a marked weight reduction in response to treatment with LIRA 
compared with treatment with metformin. Another randomized study conducted on obese women with PCOS demonstrated that short-term combined treatment with low-dose LIRA and metformin was associated with significant weight loss in obese women with PCOS who showed an inadequate response to metformin alone and lifestyle intervention (12). However, the inclusion of patients who responded poorly to metformin did not provide sufficiently appropriate controls to infer conclusive results regarding the additive effect of metformin and LIRA treatment in PCOS.

As a monotherapy, metformin did not reduce body weight, although some reports have suggested it has a modest weight lowering potential in PCOS, particularly when used as an adjunct to lifestyle intervention (13-16). However, the beneficial effects of metformin mediated via the improvement of insulin sensitivity and direct action at the ovarian level are well-established in PCOS (7). Aside from its action on classic insulin-sensitive tissue, metformin also exerts its beneficial metabolic action in part via modulation of the incretin axis, including not only the stimulatory effect of GLP-1, but also the enhancement of the expression of GLP-1 receptors and associated insulinotropic receptors (17-20).

A multi-targeted approach focusing simultaneously on modifiable weight-dependent and independent determinants of abnormalities should be considered early in the treatment management of obese patients with PCOS. It was hypothesized that the synergistic effect of metformin mediated via the modulation of the incretin axis could significantly enhance the weight-lowering potential of LIRA. The aim of the present randomized study was to evaluate whether the combination of metformin and low-dose LIRA decreased weight more effectively compared with low-dose LIRA alone in the treatment naïve obese women with PCOS.

\section{Subjects and methods}

Study design. The present study had a 12 -week prospective randomized open-label design, and was conducted with 44 obese women with PCOS (mean age, 30.3 \pm .4 .4 years; BMI, $37.2 \pm 4.5 \mathrm{~kg} / \mathrm{m}^{2}$ ) diagnosed according to the American Society for Reproductive Medicine-European Society of Human Reproduction and Embryology (ASRM-ESHRE) Rotterdam criteria (7), and recruited from the University Medical Centre (Ljubljana, Slovenia). The study is registered on www. ClinicalTrials.gov as NCT02483299.

All subjects had PCOS phenotype A, which included the concomitant presence of a) hyperandrogenemia at either the biochemical or clinical level, b) menstrual abnormalities, and c) PCO morphology. The subjects were eligible for enrollment if they were aged $>18$ years, had yet to undergo the menopause, and were obese [body mass index (BMI) $\geq 30$ ]. The exclusion criteria were a history of carcinoma, significant cardiovascular, kidney or hepatic disease, and the use of medications known to affect reproductive or metabolic functions prior to study entry. Certain subjects did, however, use oral contraceptives as advised by their gynecologists $>6$ months prior to being recruited.

The patients were randomly assigned to two groups $(n=22)$ who received either a combined treatment with metformin and LIRA (COMBO) or treatment with LIRA alone. LIRA treatment was provided at a dose of $0.6 \mathrm{mg}$ subcutaneously injected once daily and increased to $1.2 \mathrm{mg} /$ day after 1 week. Metformin was provided at a dose of $500 \mathrm{mg}$ once daily and increased by $500 \mathrm{mg}$ every 3 days until the treatment dose reached 1,000 $\mathrm{mg}$ twice daily. In the group receiving COMBO, the patients were treated with $\geq 1,000 \mathrm{mg}$ twice daily metformin for 14 days prior to treatment with $1.2 \mathrm{mg}$ LIRA once daily. During treatment with LIRA, the patients were provided with glucose-monitoring devices (Ascensia Diabetes Care Contour $^{\circledR}$; Bayer AG, Leverkusen, Germany) and supplies and educated on their use. They were instructed to measure blood glucose levels and any signs and symptoms suggesting low blood glucose levels. Hypoglycemia was defined according to the American Diabetes Association criteria as symptoms suggestive of low blood glucose confirmed by self-monitored blood glucose measurement $<3.9 \mathrm{mmol} / 1$ (21). All women in the study were instructed to report any side effects experienced during the treatment, and were given advice on the recommended lifestyle intervention that was actively promoted at the beginning of the study (22).

The primary outcome of the study was mean changes in the severity of obesity. Secondary outcomes included metabolic and hormonal changes. At the end of the study all patients underwent standard anthropometric measurements: Height, weight, waist circumference, and measurement of whole-body composition using a Hologic Dual Energy X-ray Absorptiometer (Hologic, Inc., Marlborough, MA, USA), including visceral adipose tissue (VAT) area, volume and mass. BMI was calculated as the patient weight $(\mathrm{kg}) /$ height $\left(\mathrm{m}^{2}\right)$. Prior to randomization, transvaginal ultrasound scans of the ovaries were performed by an experienced sonographer. The presence of PCO morphology was determined by the presence of $\geq 12$ follicles in each ovary measuring 2-9 $\mathrm{mm}$ in diameter and/or increased ovarian volume $\left(>10 \mathrm{~cm}^{3}\right)$.

Blood was obtained from the women during fasting to determine the levels of glucose, insulin, luteinizing hormone (LH), follicle-stimulating hormone (FSH), androstenedione and total and free testosterone, and a standard $75 \mathrm{~g}$ oral glucose tolerance test (OGTT) was used to assess glucose homeostasis. Glucose levels were determined using a glucose analyzer (SensoStar GL30 touch and GL one; DiaSys Diagnostic Systems $\mathrm{GmbH}$, Holzheim, Germany) with a standard glucose oxidase method (Beckman Coulter, Inc., Brea, CA, USA). Insulin levels were determined using an Immulite 2000 solid-phase enzyme-labeled chemiluminescent immunoradiometric assay (Siemens Healthcare, Erlangen, Germany). LH and FSH levels were determined using an Immulite 2000 solid-phase two-site chemiluminescent immunometric assay (Siemens Healthcare). Androstenedione levels were measured by specific double antibody radioimmunoassay (RIA) (Beckman Coulter) using ${ }^{125}$ I-labeled hormones (Diagnostic Systems Laboratories, Webster, TX, USA). Total and free testosterone levels were measured by coated tube RIA (DiaSorin, Salluggia, Italy and Siemens Healthcare, respectively). Sex hormone-binding globulin (SHBG) levels were determined using a chemiluminescent immunoassay (Immulite 2000 Analyzer; Siemens Healthcare). Intra-assay variations ranged from 1.6 to $6.3 \%$, and inter-assay variations ranged from 5.8 to $9.6 \%$ for the applied methods. Pre and post-treatment samples from each patient were assayed in the same assay run. 
Homeostasis model assessment (HOMA) calculation was applied as a measure for insulin resistance (IR) (23). Safety clinical assessment was performed at the beginning and at weeks 4, 8 and 12 of the treatment period, and involved the reporting of all transient or intermittent adverse avents. Pregnancy was excluded by measuring the levels of $\beta$-human chorionic gonadotropin (HCG) using an Immulite HCG solidphase two-site chemiluminescent immunometric assay in the serum (Siemens Healthcare). Women were advised to strictly use barrier contraception.

Ethical approval. The present study was approved by the Slovenian National Medical Ethics Committee and conducted in accordance with the Declaration of Helsinki and Clinical Practice guidelines (7). Written informed consent was obtained from all patients prior to participation.

Statistical analysis. Sample size was determined based on mean change in weight and data from previous studies that used comparative treatment intervention with Power and Sample Size Calculation software, version 3.0 .43 (24). To detect a statistically significant difference between groups of $\sim 2.5$-kg weight loss with $80 \%$ power, it was necessary for each group to consist of 22 patients.

The data are presented as the mean \pm standard deviation (SD). Non-parametric Wilcoxon signed-rank test for associated samples was used to compare the pretreatment and post-treatment values of continuous variables in each of the treatment groups, and a McNemar test was used for categorical variables. To compare changes in clinical parameters among the various treatment groups, a non-parametric Mann-Whitney test was used. For comparison of the distribution of responders and non-responders, logistical regression was used, and odds ratios (ORs) and 95\% confidence intervals (CI) were calculated. $\mathrm{P}<0.05$ was considered to indicate a statistically significant result. All statistical analyses were performed using SPSS software version 19.0 (IBM Corporation, Armonk, NY, USA).

\section{Results}

Baseline results. The present study included 44 subjects. One of the patients who received LIRA treatment alone discontinued the study due to protocol violation. The remaining 43 patients (aged 30.3 \pm 4.4 years; BMI $37.2 \pm 4.5 \mathrm{~kg} / \mathrm{m}^{2}$; mean $\pm \mathrm{SD}$ ) completed the study. A total of 22 patients were treated with COMBO and 21 with LIRA alone. The baseline characteristics of the patients are presented in Table I.

Measures of obesity. The mean post-treatment measurement of obesity are presented in Table I. At the end of the study, weight, BMI, waist circumference, VAT area, mass and volume were significantly decreased in all patients (all $\mathrm{P}<0.001)$. Significant weight and BMI reductions compared with pretreatment values were also observed in patients treated with LIRA alone or COMBI (all $\mathrm{P}<0.001$ ). Both LIRA alone and $\mathrm{COMBI}$ treatments also resulted in a significant reduction in waist circumference $(\mathrm{P}=0.002$ and $\mathrm{P}<0.001$, respectively), VAT mass $(\mathrm{P}=0.002$ and $\mathrm{P}=0.008$, respectively), VAT volume $(\mathrm{P}=0.002$ and $\mathrm{P}=0.009$, respectively $)$ and VAT area $(\mathrm{P}=0.002$ and $\mathrm{P}=0.009$, respectively).
Subjects treated with COMBO lost on average $6.2 \pm 2.4 \mathrm{~kg}$ compared with a $3.8 \pm 3.5-\mathrm{kg}$ weight loss in the LIRA group $\left(\mathrm{P}=0.024\right.$; Table II). BMI decreased by $2.2 \pm 0.8 \mathrm{~kg} / \mathrm{m}^{2}$ in the COMBO group compared with $1.4 \pm 1.2 \mathrm{~kg} / \mathrm{m}^{2}$ in the LIRA group $(\mathrm{P}=0.024)$. Clinically significant $(\geq 5 \%)$ weight reduction was achieved in 13 of the patients in the COMBO group (59.1\%), and 9 of the patients in the LIRA group (42.9\%; OR=1.93, 95\% $\mathrm{CI}=0.57-6.47)$; however, the difference between the treatment groups was not statistically significant $(\mathrm{P}=0.289)$.

Although waist circumference decreased by $12 \pm 20.2 \mathrm{~cm}$ in patients treated with $\mathrm{COMBO}$ and only $5.5 \pm 6.8 \mathrm{~cm}$ in patients treated with LIRA alone, the difference was not statistically significant $(\mathrm{P}=0.278)$. No statistical differences in the reduction of VAT were observed between the treatment groups (Table II).

Metabolic parameters. In all patients, significant reductions in fasting glucose levels $(\mathrm{P}=0.003)$, glucose levels at $120 \mathrm{~min}$ of OGTT $(\mathrm{P}=0.003)$ and HOMA-IR $(\mathrm{P}=0.032)$ were observed at the end of the study (Table I). Significant reductions in glucose levels at 0 and $120 \mathrm{~min}$ of OGTT were only observed in the COMBI group $(\mathrm{P}=0.001$ and $\mathrm{P}=0.002$, respectively $)$ and not in the LIRA group.

Fasting glucose decreased by $0.7 \pm 1.1 \mathrm{mmol} / 1$ in patients treated with COMBO, but only by $0.1 \pm 0.6 \mathrm{mmol} / 1$ in patients treated with LIRA, although the difference did not reach statistical significance $(\mathrm{P}=0.051)$. COMBI treatment was significantly more effective than LIRA treatment in reducing glucose as determined by $120-\mathrm{min}$ OGTT $(\mathrm{P}=0.031)$. The difference in the reductions of HOMA-IR was not statistically significant $(\mathrm{P}=0.735$; Table II).

In all patients combined, at the beginning of the study 20 subjects $(46.5 \%)$ exhibited metabolic syndrome [as determined by the criteria of the International Diabetes Federation (25)] compared with 11 subjects (25.6\%) following treatment, which was statistically significant $(\mathrm{P}=0.022)$. In the COMBI group, a significantly greater percentage of women exhibited a reversion of metabolic syndrome compared with women treated with LIRA alone [12 women $(54.5 \%)$ at the beginning of the study, vs. 6 women $(27.3 \%)$ at the end of the study in the COMBI group, as compared with 8 women $(38.1 \%)$ at the beginning of the study, vs. 5 women $(23.8 \%)$ at the end of the study in the LIRA group]; the difference within each treatment group was not statistically significant $(\mathrm{P}=0.070$ and $\mathrm{P}=0.375$, respectively).

Endocrine parameters. In all patients combined, significant reductions in androstenedione $(\mathrm{P}=0.025)$, and total $(\mathrm{P}=0.013)$ and free $(\mathrm{P}=0.001)$ testosterone, as well as significant increases in SHBG $(\mathrm{P}<0.001)$ were observed at the end of the study. However, although significant differences were observed in the COMBI group for all of these parameters, only free testosterone and SHBG levels changed significantly in patients treated with LIRA alone (Table I). No statistically significant differences were observed in LH or FSH levels. The only significant difference between the two treatment groups in endocrine parameters was observed for androstenedione levels, of which the reduction was significantly greater in patients treated with COMBI, as compared with patients treated with LIRA alone $(2.3 \pm 2.9 \mathrm{nmol} / 1$, vs. $0.0 \pm 2.3 \mathrm{nmol} / 1$, $\mathrm{P}=0.029$; Table II). 


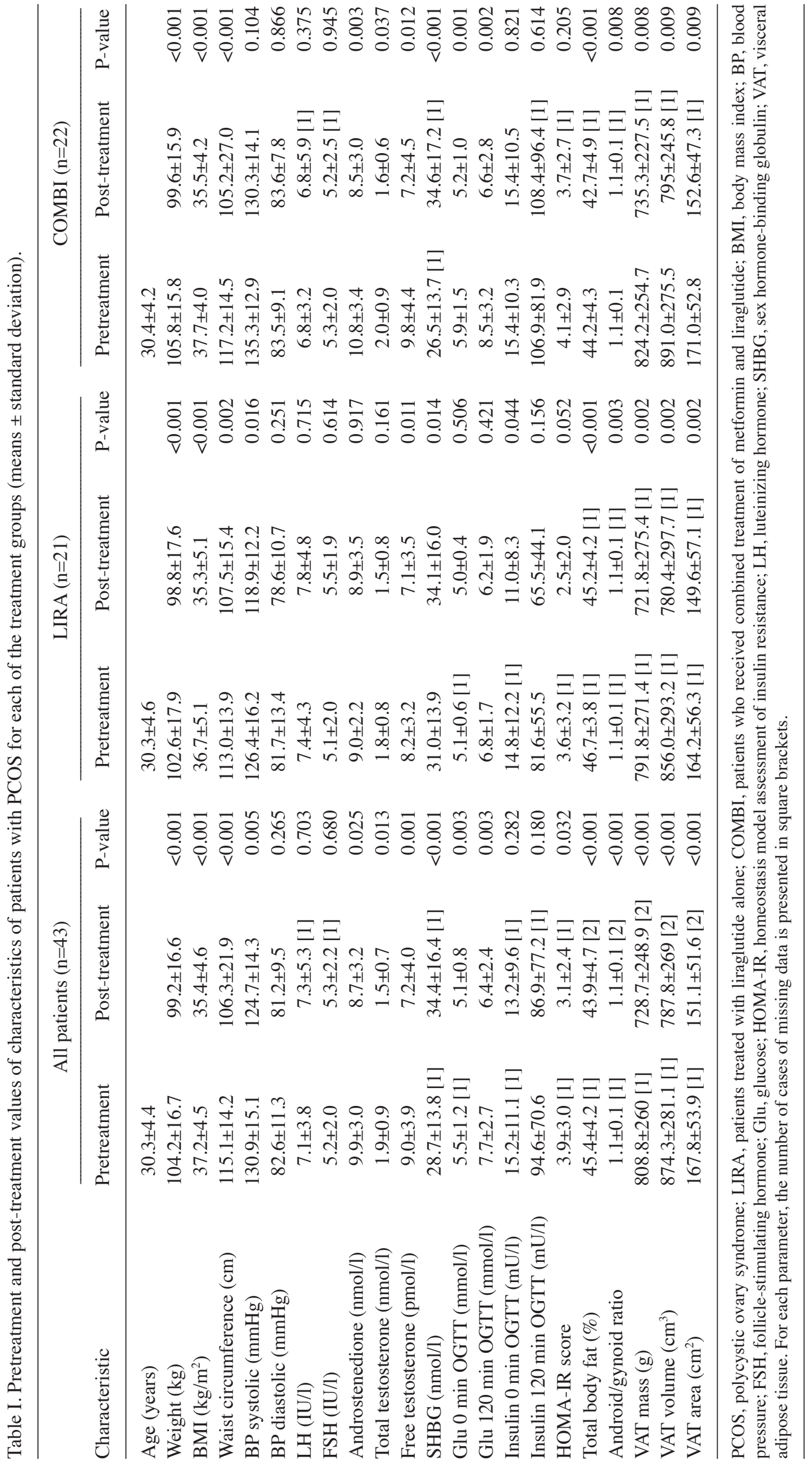


Table II. Comparison of the observable characteristics in patients with PCOS between the two treatment groups.

Absolute change (mean \pm standard deviation)

\begin{tabular}{|c|c|c|c|}
\hline Characteristic & $\operatorname{LIRA}(\mathrm{n}=21)$ & COMBI $(n=22)$ & P-value \\
\hline Weight (kg) & $-3.8 \pm 3.5$ & $-6.2 \pm 2.4$ & 0.024 \\
\hline $\operatorname{BMI}\left(\mathrm{kg} / \mathrm{m}^{2}\right)$ & $-1.4 \pm 1.2$ & $-2.2 \pm 0.8$ & 0.024 \\
\hline Waist circumference (cm) & $-5.5 \pm 6.8$ & $-12 \pm 20.2$ & 0.278 \\
\hline BP systolic (mmHg) & $-7.5 \pm 12.8$ & $-5 \pm 13.5$ & 0.559 \\
\hline BP diastolic (mmHg) & $-3 \pm 13.1$ & $0.1 \pm 6.5$ & 0.135 \\
\hline LH (IU/1) & $0.4 \pm 3.8$ & $0 \pm 5.1$ & 0.359 \\
\hline FSH (IU/l) & $0.4 \pm 2.2$ & $0.2 \pm 2.6$ & 0.589 \\
\hline Androstenedione (nmol/l) & $0 \pm 2.3$ & $-2.3 \pm 2.9$ & 0.029 \\
\hline Total testosterone (nmol/l) & $-0.3 \pm 1$ & $-0.4 \pm 0.8$ & 0.679 \\
\hline Free testosterone (pmol/l) & $-1 \pm 1.5$ & $-2.5 \pm 4.3$ & 0.074 \\
\hline SHBG (nmol/1) & $3.1 \pm 5.2$ & $8.1 \pm 9$ & 0.112 \\
\hline Glu 0 min OGTT (mmol/l) & $-0.1 \pm 0.6$ & $-0.7 \pm 1.1$ & 0.051 \\
\hline Glu $120 \mathrm{~min}$ OGTT (mmol/l) & $-0.4 \pm 1.6$ & $-2 \pm 2.6$ & 0.031 \\
\hline Insulin 0 min OGTT (mU/l) & $-3.6 \pm 7.7$ & $-0.2 \pm 6.2$ & 0.088 \\
\hline Insulin $120 \mathrm{~min}$ OGTT (mU/l) & $-16.1 \pm 44.4$ & $0.5 \pm 85.7$ & 0.811 \\
\hline HOMA-IR score & $-0.9 \pm 2.3$ & $-0.6 \pm 1.8$ & 0.735 \\
\hline Total body fat (\%) & $-1.4 \pm 1.4$ & $-1.6 \pm 1.2$ & 0.349 \\
\hline Android/gynoid ratio & $0 \pm 0$ & $0 \pm 0$ & 0.633 \\
\hline VAT mass (g) & $-73.6 \pm 90.8$ & $-64.9 \pm 104.6$ & 0.924 \\
\hline VAT volume $\left(\mathrm{cm}^{3}\right)$ & $-79.5 \pm 98.4$ & $-70 \pm 113.3$ & 0.924 \\
\hline VAT area $\left(\mathrm{cm}^{2}\right)$ & $-15.4 \pm 19$ & $-13.4 \pm 21.7$ & 0.892 \\
\hline
\end{tabular}

PCOS, polycystic ovary syndrome; LIRA, patients treated with liraglutide alone; COMBI, patients who received combined treatment of metformin and liraglutide; BMI, body mass index; BP, blood pressure; FSH, follicle-stimulating hormone; Glu, glucose; HOMA-IR, homeostasis model assessment of insulin resistance; LH, luteinizing hormone; SHBG, sex hormone-binding globulin; VAT, visceral adipose tissue. Statistically significant results are presented in bold.

Adverse events. Reporting of all transient or intermittent adverse events and the precise duration of the symptoms were included in the study protocol. The most commonly reported adverse events in the LIRA group were nausea $(6 / 21)$ and diarrhea (6/21). Vomiting was documented in $1 / 22$ patients, and episodes of mild headache $(2 / 21)$ were reported. All side effects resolved within the first 4 weeks. Adverse events associated with COMBI treatment were nausea (8/22), diarrhea (5/22), headache (1/22) and insomnia $(1 / 22)$. The majority of these side effects were present in the first month of treatment, and in the second month of treatment 2/22 mild cases of diarrhea were reported. Nausea in both groups was present for up to three days when LIRA was initiated at a dose of $0.6 \mathrm{mg}$ injected subcutaneously once daily, and if present reappeared for 2-3 days when the dose was increased to $1.2 \mathrm{mg}$ /day following one week. Nausea was not accompanied by vomiting in all but 1 patient. A single hypoglycemic episode was reported in 1 woman undergoing LIRA treatment alone, and in 2 women undergoing COMBI treatment. Some subjects in all treatment groups had multiple side effects. No side effects were reported in $13 / 21$ patients in the LIRA group, and 10/22 patients in the COMBI group. The adverse effects did not lead to withdrawal from the study in either group.

\section{Discussion}

To the best of our knowledge, the present study is the first to report that metformin combined with low-dose LIRA is more effective than low-dose LIRA alone in reducing mean body weight following 12 weeks in treatment naïve obese women with PCOS. Treatment with metformin increased the number of individuals achieving a clinically significant $\geq 5 \%$ weight loss to $\sim 60 \%$, as compared with $42.9 \%$ of patients treated with LIRA alone. Both the LIRA and COMBI treatments resulted in a significant reduction of waist circumference and VAT. The decrease in obesity-associated characteristics was correlated with beneficial effects on fasting glucose as determined by OGTT and HOMA-IR, the resolution of metabolic syndrome with reductions in androstenedione and total and free testosterone levels, as well as a significant increase in SHBG levels in the two groups. Reductions in glucose and androstenedione levels in the COMBO group were significantly greater compared with those in women treated with LIRA alone.

In a multicenter, placebo-controlled 20 week trial, treatment with LIRA led to dose-dependent weight loss that was significantly greater than that achieved with placebo at all doses (9). When compared with orlistat, only higher doses of 
2.4 and $3.0 \mathrm{mg}$ were significantly more effective in reducing body weight. Treatment with $1.2 \mathrm{mg}$ LIRA in that trial led to $4.8-\mathrm{kg}$ reduction in weight, compared with the $3.8-\mathrm{kg}$ reduction observed in the LIRA group in the present study, although the reduction in the previous trial was achieved over a 8 week-longer observation period. Also, in the previous trial, treatment with $2.4 \mathrm{mg}$ LIRA led to a $6.3 \mathrm{-kg}$ weight loss, which was similar to the $6.2-\mathrm{kg}$ reduction observed in the patients treated with COMBO in the present study. The percentage of patients who responded well and lost $\geq 5 \%$ baseline weight in the COMBO group (59.1\%) was similar to the $60.8 \%$ of patients who responded well to treatment with $2.4 \mathrm{mg}$ LIRA in the previous study, and considerably higher compared with the $52.1 \%$ of patients who responded well to treatment with $1.2 \mathrm{mg}$ LIRA in the aforementioned trial (9).

Concordant with the results of the present study, we previously demonstrated the increased efficacy of low-dose LIRA as an adjunct to metformin in weight reduction in obese women with PCOS who responded poorly to metformin (12). Following randomization, the patients were treated with either $1.2 \mathrm{mg}$ LIRA alone or $1.2 \mathrm{mg}$ LIRA combined with metformin for 12 week; the COMBO group lost on average $6.5 \mathrm{~kg}$ compared with $3.8 \mathrm{-kg}$ loss in the LIRA group (12). However, the inclusion of subjects who previously responded poorly to metformin did not provide adequate controls from which to infer significant results regarding the additive effects of metformin to the weight-lowering potential of LIRA. The present study was therefore conducted in a more statistically significant manner by including drug naïve women. Furthermore, in another 24-week study on PCOS, combined treatment with the short acting GLP-1 receptor agonist exenatide and metformin resulted in a 6-kg loss compared with a $3.2-\mathrm{kg}$ loss observed in subjects treated with exenatide alone (26). The potential additive impact of metformin on the weight-lowering potential of GLP-1 receptor agonists may be in part due to amplification of the incretin axis following GLP-1 stimulation, leading to enhancement of the expression of GLP-1 receptor and associated insulinotropic receptors via a mechanism that is dependent on peroxisome proliferator-activated receptor- $\alpha$ (17).

The present study observed reductions in fasting glucose levels and glucose levels at $120 \mathrm{~min}$ in all patients, as determined by OGTT and HOMA-IR. The reductions in fasting glucose and glucose were significant only in the COMBO group. COMBO treatment was therefore significantly more effective than LIRA treatment in reducing glucose levels, as determined by OGTT. Furthermore, COMBO treatment was more effective in reversing metabolic syndrome than was LIRA monotherapy. Reduction of hepatic glucose levels, primarily via decreased gluconeogenesis, is the main mechanism underlying metformin's glucose-lowering effects, although these have yet to be fully elucidated (27). Furthermore, some of its beneficial metabolic effects may be mediated in part via the incretin axis. Metformin significantly increased the plasma levels of GLP-1 in animal models (20), and human study showed that a single metformin dose increases the plasma levels of GLP-1 following oral glucose administration in non-diabetic patients (18). Similarly, chronic therapy with metformin specifically increased total plasma GLP-1 levels in diabetic and non-diabetic subjects (19). In addition, the combination of metformin and LIRA could potentially enhance the metabolic effects of LIRA via the same mechanisms hypothesized to underlie its synergistic effect on body weight reduction.

Furthermore, metformin is well suited for combination with LIRA in PCOS-associated obesity due to its direct effects on steroidogenesis in ovaries (28). In the present study, androstenedione, total and free testosterone levels decreased whereas SHBG levels increased in the two treatment groups, although these changes were significant in the COMBO group alone. In patients treated with LIRA only, free testosterone and SHBG levels changed significantly. The reduction of androstenedione was significantly higher in patients treated with COMBO compared with those treated with LIRA. The effects on the endocrine system following treatment with COMBO were associated with significantly increased weight loss and increased improvements in metabolic parameters in this treatment group. In addition, the existence of direct beneficial effects of metformin on the androgen profile mediated via regulation of ovarian steroidogenesis were not excluded.

LIRA has associated dose-dependent adverse effects. A phase 2 trial with doses of 1.2-3.0 mg showed that $3.0 \mathrm{mg}$ induced the highest frequency of nausea, which was observed in $~ 50 \%$ of non-diabetic obese individuals (10). In phase 3 trials, nausea was reported by $\geq 40 \%$ of individuals with type 2 diabetes mellitus on lower doses of 1.2 and $1.8 \mathrm{mg}$ (29). Adverse effects in the present study were observed in $38 \%$ of individuals in the LIRA group and $45 \%$ of individuals in the COMBO group. These side effects were transient, of mild intensity and well tolerated. Nausea occurred predominantly during the increase in metformin dosage in the COMBO group, and during the first 2 weeks of LIRA dosage increase in both treatment groups. Detailed description of adverse effects determined that these effects were transient and mild and therefore unlikely to be correlated with weight loss.

The present study had some limitations. The 12 -week observation period was too short to assess the sustainability of weight reduction and metabolic and endocrine benefits from the COMBO treatment. The number of patients in each treatment group was relatively small. Larger randomized studies of longer duration are required in order to establish treatment targets and the safety profile of the proposed treatment (metformin as an initial adjunct to low-dose liraglutide) in PCOS-associated obesity. However, the strength of the present study was the implementation of an etiologically supported strategy combining two medications that act through concomitant and synergistic mechanisms to induce more effective weight loss and other metabolic and endocrine improvements in this population.

In conclusion, metformin combined with LIRA $1.2 \mathrm{mg}$ was superior to LIRA $1.2 \mathrm{mg}$ alone in reducing body weight in treatment naïve obese women with PCOS. A higher proportion of individuals achieved $\geq 5 \%$ weight loss with metformin in addition to LIRA, as compared with LIRA monotherapy. COMBO was also superior in the improvement of diabetogenic and androgen profiles of obese women with PCOS. Although these observations are not definitive, they encourage further development of individually tailored treatment strategy focusing on a combination of drugs with distinct regulatory effects on metabolic and endocrine homeostasis in various metabolic conditions associated with obesity, particularly 
PCOS. An early multi-targeting treatment approach may improve treatment outcomes and enable the use of lower dose regimens, with improved tolerability and lower cost.

\section{Acknowledgements}

The authors of the present study are grateful for the assistance of Mirela Ozura and Elizabeta Stepanovic, registered nurses. The present study was supported by the Ministry of Health, Republic of Slovenia, and a Tertiary Care Scientific grant of the University Medical Centre Ljubljana (grant no. 20120047).

\section{References}

1.Lim SS, Davies MJ, Norman RJ and Moran LJ: Overweight, obesity and central obesity in women with polycystic ovary syndrome: A systematic review and meta-analysis. Hum Reprod Update 18: 618-637, 2012.

2. Pasquali R: Obesity and androgens: Facts and perspectives. Fertil Steril 85: 1319-1340, 2006.

3. Cattrall FR and Healy DL: Long-term metabolic, cardiovascular and neoplastic risks with polycystic ovary syndrome. Best Pract Res Clin Obstet Gynaecol 18: 803-812, 2004.

4. Lass N, Kleber M, Winkel K, Wunsch R and Reinehr T: Effect of lifestyle intervention on features of polycystic ovarian syndrome, metabolic syndrome and intima-media thickness in obese adolescent girls. J Clin Endocrinol Metab 96: 3533-3540, 2011.

5. Crosignani PG, Colombo M, Vegetti W, Somigliana E, Gessati A and Ragni G: Overweight and obese anovulatory patients with polycystic ovaries: Parallel improvements in anthropometric indices, ovarian physiology and fertility rate induced by diet. Hum Reprod 18: 1928-1932, 2003.

6. Thomson RL, Buckley JD, Noakes M, Clifton PM, Norman RJ and Brinkworth GD: The effect of a hypocaloric diet with and without exercise training on body composition, cardiometabolic risk profile and reproductive function in overweight and obese women with polycystic ovary syndrome. J Clin Endocrinol Metab 93: 3373-3380, 2008

7. Legro RS, Arslanian SA, Ehrmann DA, Hoeger KM, Murad MH, Pasquali R and Welt CK; Endocrine Society: Diagnosis and treatment of polycystic ovary syndrome: An Endocrine Society clinical practice guideline. J Clin Endocrinol Metab 98 4565-4592, 2013.

8. Vilsbøll T, Christensen M, Junker AE, Knop FK and Gluud LL: Effects of glucagon-like peptide-1 receptor agonists on weight loss: Systematic review and meta-analyses of randomised controlled trials. BMJ 344: d7771, 2012.

9. Astrup A, Rössner S, Van Gaal L, Rissanen A, Niskanen L, Al Hakim M, Madsen J, Rasmussen MF and Lean ME; NN8022-1807 Study Group: Effects of liraglutide in the treatment of obesity: A randomised, double-blind, placebo-controlled study. Lancet 374: 1606-1616, 2009.

10. Lean ME, Carraro R, Finer N, Hartvig H, Lindegaard ML, Rössner S, Van Gaal L and Astrup A; NN8022-1807 Investigators: Tolerability of nausea and vomiting and associations with weight loss in a randomized trial of liraglutide in obese, non-diabetic adults. Int J Obes (Lond) 38: 689-697, 2014.

11. Jensterle M, Kravos NA, Pfeifer M, Kocjan T and Janez A: A 12-week treatment with the long-acting glucagon-like peptide 1 receptor agonist liraglutide leads to significant weight loss in a subset of obese women with newly diagnosed polycystic ovary syndrome. Hormones (Athens) 14: 81-90, 2015.

12. Jensterle Sever M, Kocjan T, Pfeifer M, Kravos NA and Janez A: Short-term combined treatment with liraglutide and metformin leads to significant weight loss in obese women with polycystic ovary syndrome and previous poor response to metformin. Eur J Endocrinol 170: 451-459, 2014.
13. Bruno RV, de Avila MA, Neves FB, Nardi AE, Crespo CM and Sobrinho AT: Comparison of two doses of metformin (2.5 and $1.5 \mathrm{~g} /$ day) for the treatment of polycystic ovary syndrome and their effect on body mass index and waist circumference. Fertil Steril 88: 510-512, 2007.

14. Gambineri A, Patton L, Vaccina A, Cacciari M, Morselli-Labate AM, Cavazza C, Pagotto U and Pasquali R: Treatment with flutamide, metformin and their combination added to a hypocaloric diet in overweight-obese women with polycystic ovary syndrome: A randomized, 12-month, placebo-controlled study. J Clin Endocrinol Metab 91: 3970-3980, 2006.

15. Harborne LR, Sattar N, Norman JE and Fleming R: Metformin and weight loss in obese women with polycystic ovary syndrome: Comparison of doses. J Clin Endocrinol Metab 90: 4593-4598, 2005.

16. Nieuwenhuis-Ruifrok AE, Kuchenbecker WK, Hoek A, Middleton P and Norman RJ: Insulin sensitizing drugs for weight loss in women of reproductive age who are overweight or obese: Systematic review and meta-analysis. Hum Reprod Update 15: 57-68, 2009.

17. Maida A, Lamont BJ, Cao X and Drucker DJ: Metformin regulates the incretin receptor axis via a pathway dependent on peroxisome proliferator-activated receptor- $\alpha$ in mice. Diabetologia 54: 339-349, 2011.

18. Mannucci E, Ognibene A, Cremasco F, Bardini G, Mencucci A, Pierazzuoli E, Ciani S, Messeri G and Rotella CM: Effect of metformin on glucagon-like peptide 1 (GLP-1) and leptin levels in obese nondiabetic subjects. Diabetes Care 24: 489-494, 2001.

19. Mannucci E, Tesi F, Bardini G, Ognibene A, Petracca MG, Ciani S, Pezzatini A, Brogi M, Dicembrini I, Cremasco F, et al: Effects of metformin on glucagon-like peptide-1 levels in obese patients with and without Type 2 diabetes. Diabetes Nutr Metab 17: 336-342, 2004.

20. Yasuda N, Inoue T, Nagakura T, Yamazaki K, Kira K, Saeki T and Tanaka I: Enhanced secretion of glucagon-like peptide 1 by biguanide compounds. Biochem Biophys Res Commun 298: 779-784, 2002

21. American Diabetes Association: Standards of medical care in diabetes-2013. Diabetes Care 36 (Suppl 1): S11-S66, 2013.

22. Moran LJ, Pasquali R, Teede HJ, Hoeger KM and Norman RJ: Treatment of obesity in polycystic ovary syndrome: A position statement of the Androgen Excess and Polycystic Ovary Syndrome Society. Fertil Steril 92: 1966-1982, 2009.

23. Matthews DR, Hosker JP, Rudenski AS, Naylor BA, Treacher DF and Turner RC: Homeostasis model assessment: Insulin resistance and beta-cell function from fasting plasma glucose and insulin concentrations in man. Diabetologia 28: 412-419, 1985.

24. Dupont WD and Plummer WD Jr: Power and sample size calculations. A review and computer program. Control Clin Trials 11: 116-128, 1990.

25. Alberti KG1, Zimmet P and Shaw J: Metabolic syndrome - a new world-wide definition. A Consensus Statement from the International Diabetes Federation. Diabet Med 23: 469-480, 2006.

26. Elkind-Hirsch K, Marrioneaux O, Bhushan M, Vernor D and Bhushan R: Comparison of single and combined treatment with exenatide and metformin on menstrual cyclicity in overweight women with polycystic ovary syndrome. J Clin Endocrinol Metab 93: 2670-2678, 2008.

27. Hundal RS, Krssak M, Dufour S, Laurent D, Lebon V, Chandramouli V, Inzucchi SE, Schumann WC, Petersen KF, Landau BR and Shulman GI: Mechanism by which metformin reduces glucose production in type 2 diabetes. Diabetes 49: 2063-2069, 2000.

28. Palomba S, Falbo A, Zullo F and Orio F Jr: Evidence-based and potential benefits of metformin in the polycystic ovary syndrome: A comprehensive review. Endocr Rev 30: 1-50, 2009.

29. Davies MJ, Kela R and Khunti K: Liraglutide-overview of the preclinical and clinical data and its role in the treatment of type 2 diabetes. Diabetes Obes Metab 13: 207-220, 2011. 